CUAD. CONTAB. / BOCOTÁ, COLOMBIA, 16 (42): 761-779 / SEPTIEMBRE-DICIEMBRE 2015 / 761

\title{
Perspectiva crítica del valor razonable en el marco de la crisis financiera*
}

doi:10.11144/Javeriana.cc16-42.pcvr

\section{Sandra Patricia Perea-Murillo}

Contadora pública, Universidad Tecnológica del Chocó

Diego Luis Córdoba, UTCH. Magíster en administración, Universidad de Medellín y participante de la sexta cohorte del doctorado en ciencias contables de la Universidad de Los Andes, ULA, Mérida, Venezuela. Docente de tiempo completo, Universidad Tecnológica del Chocó Diego Luis Córdoba, UTCH. Coordinadora del campo investigativo de la Facultad de Ciencias Administrativas Económicas y Contables, Universidad Tecnológica del Chocó Diego Luis Córdoba, UTCH, y líder del grupo de investigación Innovación Contable.

Correo electrónico: sapapemur@hotmail.com

\footnotetext{
* Artículo de reflexión. El artículo de investigación científica se deriva de la evaluación final del seminario doctoral Tendencias investigativas del pensamiento contable, asignatura adscrita al doctorado en Ciencias Contables, Universidad de los Andes, ULA, Mérida, Venezuela. Una versión preliminar de este artículo fue presentada en el Tercer Encuentro Binacional Colombia-Venezuela, Tendencias de Investigación Contables, realizada en Bucaramanga del 6 al 7 de marzo de 2015. La autora agradece los valiosos aportes de los participantes en ese encuentro.
} 


\section{2 / VOL. 16 / NO. 42 / SEPTIEMBRE-DICIEMBRE 2015}

Resumen El presente documento hace parte de las reflexiones conceptuales Sobre la emisión del estándar de valor razonable por organismos reguladores como el FASB y el IASB, en el marco de la crisis financiera de 2008 que comenzó en Estados Unidos a causa de las burbujas inmobiliarias, que también se conocen como créditos subprime. La investigación se orienta a plantear — desde una visión crítica- la forma de medir el valor razonable y su incidencia en la crisis financiera. El estudio se enmarca en el enfoque cualitativo, basado en la revisión de literatura especializada de pensadores contemporáneos de la contabilidad, que se abordaron desde una postura fundamentada en la teoría crítica. Como conclusión, la investigación destaca el uso de niveles de entradas no observables en la determinación del valor razonable que se derivan de la complejidad de los mercados inactivos en los que la entidad que reporta utiliza supuestos internos para fijar los precios de activos y pasivos que son difíciles de comprobar en la realidad.

Palabras clave valor razonable; crisis financiera; principios de contabilidad

\section{Códigos JEL M41}

\section{Critical Perspective of Fair Value in the Context of the Financial Crisis}

\footnotetext{
Abstract This document is part of the conceptual reflections on the issuance of the fair value standard by regulatory organizations such as the FASB and IASB, in the framework of the 2008 financial crisis, which started in the United States as a result of real-estate bubbles (also known as subprime loans). The research aims to outline -from a critical perspective - the way to measure fair value and its incidence on the financial crisis. The study is framed in a qualitative approach based on the review of specialized literature, written by contemporary accounting thinkers, addressed from a standpoint based on the critical theory. As a conclusion, this research highlights the use of levels of unobservable capital inflows for the determination of
}

fair value, derived from the complexity of inactive markets where the reporting entity uses internal assumptions to fix the price of assets and liabilities which are actually difficult to verify.

Keywords fair value; financial crisis; accounting principles

\section{Perspectiva crítica do valor razoável no quadro da crise financeira}

Resumo O presente documento faz parte das reflexões conceituais Sobre emissão do padrão de valor razoável pelos organismos reguladores como o FASB e o IASB, no quadro da crise financeira de 2008 que começou nos Estados Unidos a causa das bolhas imobiliárias, mesmo conhecidas como créditos subprime. A pesquisa orienta-se a levantar —desde uma visão crítica- a forma de medir o valor razoável e sua incidência na crise financeira. O estudo aborda o enfoque qualitativo, baseado na revisão de literatura especializada de pensadores contemporâneos da contabilidade, desde uma postura fundamentada na teoria crítica. Como conclusão, a pesquisa destaca o uso de níveis de entradas inobserváveis na determinação do valor razoável que derivam da complexidade dos mercados inativos nos que a instituição que reporta utiliza supostos internos para fixar os preços de ativos e passivos difíceis de comprovar na realidade.

Palavras-chave valor razoável; crise financeira; princípios de contabilidade

\section{Introducción}

El concepto de valor razonable en las últimas décadas ha sido discutido por estudiosos de la disciplina contable, economistas, financieros, epistemólogos, políticos y los organismos en- 
cargados de emitir la normatividad y los estándares contables.

Una postura defiende el modelo de contabilidad de valor razonable por considerar que muestra el valor actual de activos o pasivos con el pasar del tiempo; mientras que la otra, se opone a la aplicación del valor razonable en contabilidad, pues manifiestan que este modelo es susceptible a manipulaciones, a diferentes interpretaciones y aplicaciones que han dado paso a múltiples escándalos financieros en diferentes sectores de la economía mundial como reflejan los estudios realizados en 2011 por Philip D. Bougen y Joni J. Young en su artículo Fair Value Accounting: Simulacra and Simulation y por Milorad Stojilković en su estudio Towards a Criticism of Fair Value Accounting.

Los organismos encargados de la emisión del modelo en contabilidad de valor razonable son, por un lado, el Consejo de Normas de Contabilidad Financiera (Financial Accounting Standards Board, FASB), que establece la normatividad contable financiera en Estados Unidos para entidades del sector privado, mediante Declaraciones de Norma de Contabilidad Financiera (Statement of Financial Accounting Standards, SFAS); $y$, por el otro, el Consejo de Normas Internacionales de Contabilidad (International Accounting Standards Board, IASB). El IASB es un organismo independiente encargado de emitir International Financial Reporting Standards (IFRS) traducido al español como Estándares Internacionales de Información Financiera y oficialmente denominado Normas Internacionales de Información FinancieraNIIF para los distintos países que deseen adoptar o adaptar la normatividad.
En el escenario descrito, los organismos reguladores buscan suscitar el interés público, mediante un único conjunto de normas contables de carácter global y de cumplimiento obligatorio, para que los estados financieros sean de alta calidad, comprensibilidad, transparencia y comparabilidad, y para que los diferentes usuarios de la información financiera en todo el mundo puedan tomar mejores decisiones económicas (IASB, 2011).

El American Institute of Certified Publics Accountants (AICPA, 2004) entiende el interés público como el bienestar colectivo de la comunidad de personas e instituciones a las que sirve la profesión; mientras que para el Consejo de Normas de Contabilidad Financiera (FASB, 2011), el interés público es servir al interés de la inversión pública en materia de contabilidad e información financiera y colectivamente; y para las firmas de auditoría como PricewaterhouseCoopers, PwC, el interés público es confianza pública, firmemente basadas en una ideología neoliberal, con el fin de crear valor por medio de la formación de capital (Baker, 2009).

De forma similar a los objetivos del IASB, el FASB (2011) busca mejorar la utilidad de la información financiera, al centrarse en las principales características de relevancia y fiabilidad, y en las cualidades de comparabilidad y consistencia, y también pretende mantener los estándares actuales para reflejar los cambios en los métodos de hacer negocios y mejorar la común comprensión de la naturaleza y los propósitos de la información en los informes financieros.

Para el logro de tales fines, entre tantas normas emitidas por el IASB y el FASB, la 
normatividad de valor razonable define de forma análoga este concepto en la NIIF 13 (IASB, 2014, párrafo 9), y el SFAS 157 (FASB, 2006, párrafo 5) precisa que el valor razonable es "el precio que sería recibido por vender un activo o pagado por transferir un pasivo en una transacción ordenada entre participantes en el mercado a fecha de medición".

Sin embargo, en el desarrollo de este constructo, vemos cómo con la aplicación de la contabilidad de valor razonable, muchas de las características que deben estar presentes en la preparación y presentación de la información financiera para que sea de alta calidad son susceptibles de manipulaciones, al crear una distorsión en la realidad representada a partir de la información, pues se busca en este caso satisfacer intereses particulares que van en contra del interés colectivo; este no es el deber ser de la norma pero se da por los vacíos que puede haber en su aplicación.

Metodológicamente, el trabajo se fundamentó en el enfoque cualitativo, de carácter documental, pues se basó en la revisión de literatura especializada en los campos de valor razonable, de las crisis financieras presentadas a partir de 2008 y del estudio de la perspectiva crítica de valor razonable. El trabajo se estructuró en cuatro secciones: la primera, referida al valor razonable y su incidencia en la crisis económica presentada desde 2008; la segunda enfatiza en la relación entre el valor razonable y la concepción de la teoría subjetiva del valor, de Carl Menger; en la tercera, se aborda la crítica al valor razonable planteada por el SFAS 157. Por último, en la cuarta, se exponen las reflexiones finales a manera de conclusio- nes, entre las que se destaca que la contabilidad de valor razonable permite utilizar niveles de entradas observables y no observables para fijar los precios de activos y pasivos. En los niveles de entradas observables se utilizan los supuestos que los participantes del mercado utilizarían para fijar los precios de activos y pasivos de manera independiente a la entidad que reporta la medición, hecho que puede ser verificado con mayor facilidad por darse en un mercado activo, mientras el uso de los niveles de entradas no observables en la determinación del valor razonable se deriva de la complejidad de los mercados imperfectos e inactivos pues la entidad que reporta utiliza supuestos internos para fijar los precios de sus activos y pasivos.

Como consecuencia de la utilización de los niveles de entradas inobservables en la determinación del valor razonable de los activos y pasivos, se produjo una alteración de los resultados de los estados financieros de las empresas, ya que estos registraban ganancias no realizadas que no correspondían a la realidad presentada en el sector inmobiliario para ese momento, lo que puso en riesgo no solo el patrimonio de las entidades financieras que otorgaron préstamos hipotecarios sino también la de los fondos de inversión que accedieron a comprar la cartera vencida.

\section{Valor razonable y su incidencia en la crisis económica}

Desde hace años, en el ámbito académico, político, normativo y económico se ha venido presentando una serie de discusiones en la con- 
tabilidad sobre la aplicación del criterio de valor razonable.

En palabras de Heiberg Andrés CastellanosSánchez (2010, pp. 270-271), la contabilidad de valor razonable "ha generado controversias en los miembros de la comunidad contable. Así pues, connotados tratadistas y profesionales de la contabilidad tienen posiciones contrarias a la aplicación de esta acepción de valor, advirtiendo que a pesar de representar un significativo avance en el transitar de la ciencia contable, deja de lado los postulados básicos de prudencia y objetividad que han caracterizado a la disciplina", porque al introducirse el cálculo de valor razonable se presenta desconfianza para los diferentes usuarios de la información financiera, debido al grado de subjetividad inserta en la determinación de los niveles de entradas como técnicas de medición aplicadas en la determinación de los precios de los activos y pasivos. Entendiéndose los niveles de entradas como los supuestos que los participantes del mercado utilizarían para fijar precios.

Esta postura se evidencia en el planteamiento de Milorad Stojilković (2011, p. 92) cuando afirma que "el desplome de los mercados financieros reveló que la contabilidad del valor razonable no era un atributo de medición objetiva de las posiciones de los estados financieros" y que "la crisis financiera fue la ocasión para el examen de la contabilidad de valor razonable".

En tal sentido, destaca que los resultados financieros obtenidos mediante la contabilidad de valor razonable contienen ganancias no realizadas, y al registrarlas se atenta contra uno de los principios más antiguos en contabilidad: el conservadurismo, que busca que la información contenida en los estados financieros no esté sobreestimada para no generar expectativas falsas, que conduzcan a una inadecuada toma de decisiones de los usuarios de la información financiera; por el contrario, este principio establece la conveniencia de contabilizar las pérdidas y las ganancias solo en el momento en que ocurran. Por eso, para Stojilković (2011), resulta de gran peligro la aplicación de la contabilidad de valor razonable en el caso de las corporaciones y todos los demás interesados, porque la revelación y distribución de la ganancia no realizada pone en riesgo el capital de la empresa y su supervivencia.

De ahí que las crisis financieras que se han presentado en las últimas décadas en el mundo hayan permitido que se vuelva a debatir sobre los principios de objetividad, conservadurismo, prudencia, representación fiel, neutralidad y comparabilidad, como elementos fundamentales para la elaboración y presentación de los estados financieros, lo cual se evidencia en los estudios realizados por Philip D. Bougen y Joni J. Young (2011) así como por Milorad Stojilković (2011) e Ignacio Cabeza del Salvador (2009).

La crisis financiera trascendente que comenzó en Estados Unidos en el sector inmobiliario en 2008, se debió a que los bancos otorgaron créditos hipotecarios a personas insolventes que no tenían la capacidad de sufragar esa obligación una vez adquirida, lo que dio lugar a las burbujas inmobiliarias de crédito, conocidas como la crisis de las hipotecas subprime.

Ante el alto riesgo que representaba el otorgamiento de préstamos hipotecarios a personas con escasa solvencia, la banca cobró intereses 


\section{6 / VOL. 16/ NO. 42 / SEPTIEMBRE-DICIEMBRE 2015}

más altos. Además, algunas compañías inversoras no podían darse cuenta del riesgo inserto en los créditos hipotecarios entregados, pues confiaban en una información contable que no reflejaba fielmente la realidad.

Como consecuencia, los bancos procedieron a transformar los créditos en acciones para obtener liquidez y como forma de capitalizarse, comenzaron a realizar las transacciones mediante la titularización (securitization ${ }^{1}$ ) de créditos hipotecarios con fondos de inversión; las entidades bancarias disfrutaron de la posibilidad de retirar estos pasivos del balance y trasladar los riesgos de incobrabilidad de los créditos hipotecarios a los fondos, que tenían como garantía las viviendas y las expectativas de que el precio de esas casas se incrementara.

Esta situación dio lugar a muchos escándalos financieros, ya que los bancos registraban sus activos en el balance por encima de su valor real de mercado, por el hecho de que el criterio de valoración en contabilidad de valor razonable consiente la utilización de entradas inobservables - que profundizaremos más adelante-, lo que permitió que las entidades en mención sobrevaloraran sus activos y los convirtieran en activos que no reflejaban su valor real.

En este escenario, se generó una crisis financiera para las entidades que compraron títulos hipotecarios, cuando los precios de las viviendas dejaron de subir y, por el contrario, comenzaron a bajar, como consecuencia de que la reserva federal decidió subir las tasas de intereses para los compradores, generándose una situación donde

1 Consiste en la transformación de préstamos otorgados por una entidad financiera en títulos valores ofertados entre el público inversor. una parte importante de los deudores de las viviendas dejaron de pagar la hipoteca.

Muchas instituciones fueron absorbidas por otras, entre ellas Merrill Lynch absorbida por Bank of America, que tuvo que pagar alrededor de US\$20.000 millones de dólares para asumir las pérdidas por la compra de esta institución; por su parte, Lehman Brothers, Bear Stearns, Northern Rock, etc., se declararon en quiebra y fueron absorbidas por el Estado (Sánchez-Salamanca, 2009).

En tal sentido, Leonor Delgado-Piña (2011, p. 75) señala:

Son muchos los que señalan a la aplicación del criterio del valor razonable en la valoración de los activos y pasivos, como "culpable", en parte, de la crisis económica, por agudizar los efectos de la misma. Esta asociación se debe a que la aplicación del valor razonable en épocas de recesión o crisis produce pérdidas, poniendo a las empresas en una situación económico-financiera complicada. Y es que ante una situación de caídas de los precios continuadas, se incrementa la incertidumbre sobre si los precios o parámetros que se obtienen en el mercado son fiables.

Este hecho generó mayor complejidad en la aplicación y cumplimiento de los Principios de Contabilidad Generalmente Aceptados, emitidos por FASB (2011) en diferentes entes económicos, no solo en Estados Unidos, sino también en Europa y otros lugares del mundo, pues se vieron afectados algunos principios de contabilidad como la objetividad, el conservadurismo o prudencia y la uniformidad. 
En igual medida, con la aplicación del valor razonable por las entidades financieras, las características cualitativas y cuantitativas de la información financiera emitidas por el IASB, se vieron vulneradas; entre estas resulta pertinente mencionar: la relevancia, la fiabilidad, la comparabilidad y la comprensibilidad.

Al respecto, Milorad Stojilković (2011) considera que la relevancia de la información financiera fue transgredida con la aplicación del valor razonable, porque según el Marco conceptual para la información financiera (IASB, 2011) "la información financiera relevante es capaz de influir en las decisiones tomadas por los usuarios" (A. 33). En tal orientación, Stojilković manifiesta que la aplicación consistente del valor razonable produce información relevante para que los usuarios de la información financiera puedan tomar decisiones, por proporcionar tanto el pronóstico como la verificación de esta información pero solo en el papel, en vista de que los mercados no son estables ni eficientes.

Por ello, Stojilković (2011, p. 104) considera que con la aplicación del valor razonable:

En la valoración de los activos financieros, las inversiones mobiliarias e inmobiliarias, como también de los pasivos, han revelado que no es posible valorar con precisión el futuro valor razonable de los activos netos sobre la base de la hoja de balance, como tampoco los resultados financieros futuros con base en los estados de pérdidas y ganancias.

Tal situación se presenta como consecuencia de que la contabilidad de valor razonable no ofrece información relevante para los usua- rios de la información financiera, por no tener la capacidad de dar cuenta de las condiciones de expansión de los mercados, de las crisis que se presentan en estos, y de las oscilaciones frecuentes experimentadas en los mercados en el corto plazo a causa de la especulación, entre otras razones (Stojilković, 2011).

Analógicamente, la comparabilidad como característica cualitativa de la información financiera emitida por el FASB (2011) también difiere en su aplicación práctica, debido a que hay muchos ejemplos de activos que son idénticos según la norma, pero por utilizar diferentes técnicas (de mercado, de ingresos o resultados y de costo) para la determinación del valor razonable, resulta improbable la comparación del activo, ya que -implícitamente- puede haber factores de tiempo y espacio que son considerados para la medición del activo, lo cual no se refleja en el modelo utilizado para esta valoración.

Esta situación da lugar al incumplimiento del objetivo central de la información financiera: ser útil para la toma de decisiones a los distintos usuarios de la información en diferentes partes del mundo (IASB, Marco conceptual, 2011).

Por su parte, Robert H. Herz, presidente del comité de las Normas de Contabilidad Financiera, declaró el 29 de marzo de 2006 ante el U.S. House of Representatives Financial Services Subcommittee On Capital Markets, Insurance, and Government Sponsored Entities, antes de emitir el SFAS 157 de valor razonable y mediciones publicado en septiembre de 2006. En esa comparecencia, Herz opinó que la complejidad que impregna los sistemas para la preparación y presentación de los informes que se evidencia por el volumen y el detalle de la contabilidad, 
auditoría, normas y regulación, pone un reto importante para mantener y mejorar la exactitud y transparencia de la información financiera presentada a los inversores y acreedores. Y reconoce que la complejidad de la presentación de información financiera basada en NIIF ha generado cambios en el enfoque contable, la profesión y la auditoría, ya que cada vez más es necesaria la participación de expertos técnicos para garantizar su cumplimiento.

Años más tarde, con la crisis global, Robert H. Herz (2009) recordó a los que culparon al valor razonable de la crisis, que el SFAS 157 no es un concepto nuevo y que su objeto era "establecer una definición coherente de valor razonable. También proporcionar un marco coherente para determinar el valor razonable de diversos tipos de activos y pasivos" (Herz, 2009 , p. 4), lo que es nuevo ahora son todos los problemas que constituyeron la crisis como:

Los préstamos notoriamente laxos y fraudulentos, el exceso de apalancamiento, la creación de inversiones complejas y arriesgadas mediante la titularización y los derivados, la distribución mundial de este tipo de instrumentos con rápido crecimiento no regulado y los mercados opacos que carecen de una infraestructura adecuada para los mecanismos de compensación y de descubrimiento de precios, las clasificaciones erróneas, y la ausencia de procesos adecuados de gestión de riesgos y valoración en muchas instituciones financieras (Herz, 2009, p. 5).

Ante este panorama, el FASB se negó a reconocer los riesgos y la culpabilidad de la apli- cación de técnicas de valoración como el valor razonable en la quiebra de muchos bancos como responsables de la crisis financiera de 2008. Pero es evidente que el papel que juega la contabilidad cada vez se hace más difícil de entender, ya que no está claro si la contabilidad además de dar cuenta sobre hechos pasados y del presente para la toma de decisiones, debe tomar una posición predictiva, como lo preceptúa la NIF A 4, características cualitativas de la información financiera, que afirma que la información financiera es relevante cuando:

Influye en la toma de decisiones económicas de quienes la utilizan. Para que la información sea relevante debe: a) servir de base en la elaboración de predicciones y en su confirmación (posibilidad de predicción y confirmación); y b) mostrar los aspectos más significativos de la entidad reconocidos contablemente (importancia relativa) (FASB, NIF A 4, 2006, p. 6).

Y entiende que la posibilidad de predicción y confirmación de la información financiera debe contener:

\footnotetext{
Elementos suficientes para coadyuvar a los usuarios generales a realizar predicciones; asimismo, debe servir para confirmar o modificar las expectativas o predicciones anteriormente formuladas, permitiendo a los usuarios generales evaluar la certeza y precisión de esta información (FASB, NIF A 4, 2006, p. 6).
}

Hechos como estos abren paso a la discusión que se ha dado en contabilidad desde hace 
años sobre la importancia de la contabilidad a costo histórico, ya que teóricos muy destacados en la disciplina contable defienden este modelo. Por ejemplo, Ananias Charles Littleton (1955) afirma que el modelo al costo histórico "desde siempre ha gozado de mayor privilegio, por el hecho de que los datos básicos en contabilidad no son ni muestras, ni promedios, sino datos originales acordados por las partes que participan en una transacción" (p. 365). Además, este método de valoración no tiene en cuenta estimaciones $y$, por lo tanto, se convierte en un método de valoración que proporciona un alto nivel de objetividad, es más prudente y se aproxima mejor al valor real (Robert Sterling, 1967, en Josephine Maltby, 2000, p. 52).

En concordancia con lo anterior, Yuji Ijiri (1975) considera que el costo histórico es pertinente para la toma de decisiones económicas, debido a que proporciona datos que son menos discutibles que los proporcionados por otros métodos de valuación propuestos usualmente como requisito para una contabilidad financiera. Y explica que la objetividad del criterio de valuación al costo histórico está dada por su fácil verificación, basada en transacciones reales, estando en contra de la subjetividad y de la manipulación de otros criterios valorativos entre los que se encuentran el costo corriente o el precio de venta.

En contraposición a lo anterior, el profesor Richard Mattessich (2002, p. 163), uno de los más destacados investigadores de la ciencia contable del siglo XX, en su libro Contabilidad y métodos analíticos, manifiesta que el principio del costo puede, hablando legalmente, "ser más objetivo y puede evitar algún tipo de incerti- dumbre, pero al hacerlo viola uno de los componentes fundamentales de cualquier teoría del valor; el que el valor de un objeto o hecho se halla atado a circunstancias de tiempo". En consecuencia, un valor determinado constituye una magnitud moderada y altamente inestable.

\section{Relación del valor razonable y la teoría subjetiva del valor}

Los modelos de regulación contable son propuestos por organismos como el IASB, que emite las Normas Internacionales de Información Financiera y el FASB, órgano encargado de emitir para el sector privado las normas de contabilidad y de reporte financiero en Estados Unidos (SFAS). En frases del profesor Harold ÁlvarezÁlvarez (2009), se considera que la normatividad contable se fundamenta de forma parcial en la teoría neoclásica del valor, al establecer criterios valorativos basados en el valor razonable como el "valor justo" para los diversos negociadores y usuarios.

Esto dio lugar a una importante reflexión fundamentada en las concepciones de Carl Menger (1871), reconocido economista neoclásico, nacido en Nowy Sacz, Galitzia, en el Imperio austríaco (actual Polonia), padre del pensamiento económico de la Escuela Austríaca, porque puso en duda la teoría clásica del valor basado en los costos de producción, planteada por Adam Smith y David Ricardo, al contemplar que el precio de un producto o servicio no se crea siguiendo la suma de todos sus costos más el margen del empresario, sino que al producto se le asigna un precio en el mercado (valor razonable) de acuerdo a 


\section{0 / VOL. 16 / NO. 42 / SEPTIEMBRE-DICIEMBRE 2015}

las necesidades humanas y a partir de aquí se moldean sus costos.

En otras palabras, Carl Menger planteó un mecanismo de fijación de precios, basado en el equilibrio entre la oferta y la demanda $y$, por ende, en los valores que da el mercado de acuerdo a las necesidades de compradores y vendedores, como para muchos casos estos valores de mercados son requeridos para la determinación del valor razonable de un activo.

En cuanto a la medición del valor razonable, Richard Mattessich (2002, p. 147) advierte que "1) los precios de mercado están sujetos a aberraciones monetarias, 2) pueden ser manipulados, y 3) no siempre tienen validez mundial". Por tanto, considerar el valor de mercado como un valor objetivo-justo, no contempla la noción de objetividad empleada en la ciencia.

Con respecto a la objetividad del valor, Carl Menger (1871) dilucida:

Es erróneo objetivar el concepto de "valor" debido a que el valor no es algo inherente a los bienes, no es una cualidad intrínseca de los mismos, ni menos aún una cosa autónoma, independiente, al contrario es un juicio que se forman los hombres sobre la significación que tiene la posesión del bien para la conservación de su vida o, respectivamente, de su bienestar $y$, por ende, no existe fuera del ámbito de su conciencia. Por lo tanto lo único objetivo son las cosas o, respectivamente, las cantidades de cosas, y su valor es algo esencialmente distinto de ellas. La objetivación del valor de los bienes, que es por su propia naturaleza totalmente subjetivo (p. 86).
La discusión acerca de la subjetividad del valor radica en que los activos adquieren mayor o menor valor dependiendo de la importancia que el bien tenga para el sujeto o por el grado de utilidad que el activo tiene en la satisfacción de sus necesidades humanas, lo que quiere decir que lo que vale muy poco para unos, puede valor mucho para otros.

De allí que Carl Menger (1871) para fundamentar la teoría subjetiva del valor haya utilizado el enfoque individualismo metodológico, el cual sostiene que todos los fenómenos sociales, estructurales y de cambios son en principio explicables por elementos individuales, es decir, del sujeto y niega que una colectividad sea un organismo que toma decisiones autónomas, dejando de lado sus sesgos e intereses, por cuanto el valor de un bien (activo) o un servicio no es neutral.

Y aun así, con estas consideraciones, se observa que la normatividad contable les resta importancia a estas bases teóricas al abordar la realidad contable desde una perspectiva objetiva, que excluye la subjetividad implícita en el concepto de valor.

En concordancia con el anterior argumento, Norman Macinstosh (2009) citado por Norka Viloria-Ortega (2013, p. 46), "propone desmontar los supuestos del realismo arraigados en la contabilidad, expresados en el supuesto de que los hechos económicos existen independientemente del sujeto y de su representación por la contabilidad".

Norman B. Macintosh, Teri Shearer, Daniel B. Thornton y Michael Welker (2000) consideraron que la representación contable hoy nada tiene qué ver con la realidad, debido a que se 
remite a la ausencia de la realidad en los estados financieros, es decir, en los estados financieros se simula lo que no se tiene o se crea lo que le convenga a la gerencia del ente económico, apartándose del principio de representación fiel y moviéndose así la contabilidad en un mundo de hiperrealidad ${ }^{2}$.

\section{Críticas a SFAS 157 de valor razonable}

En octubre de 2004, el IASB y el FASB emprendieron un proyecto conjunto para desarrollar un marco conceptual y estándares comunes para las prácticas de reportaje de contabilidad formales. En mayo de 2006, el FASB emitió la norma SFAS 157: medición a valor razonable, que en conjunto con el IASB, organismo emisor de normas contables, busca unificar los requisitos y criterios para la medición a valor razonable.

A continuación, se detalla la estructura de los eventos realizados por IASB y FASB para emitir la norma de valor razonable, con miras a tener estándares comunes para la elaboración y presentación de la información financiera (tabla 1).

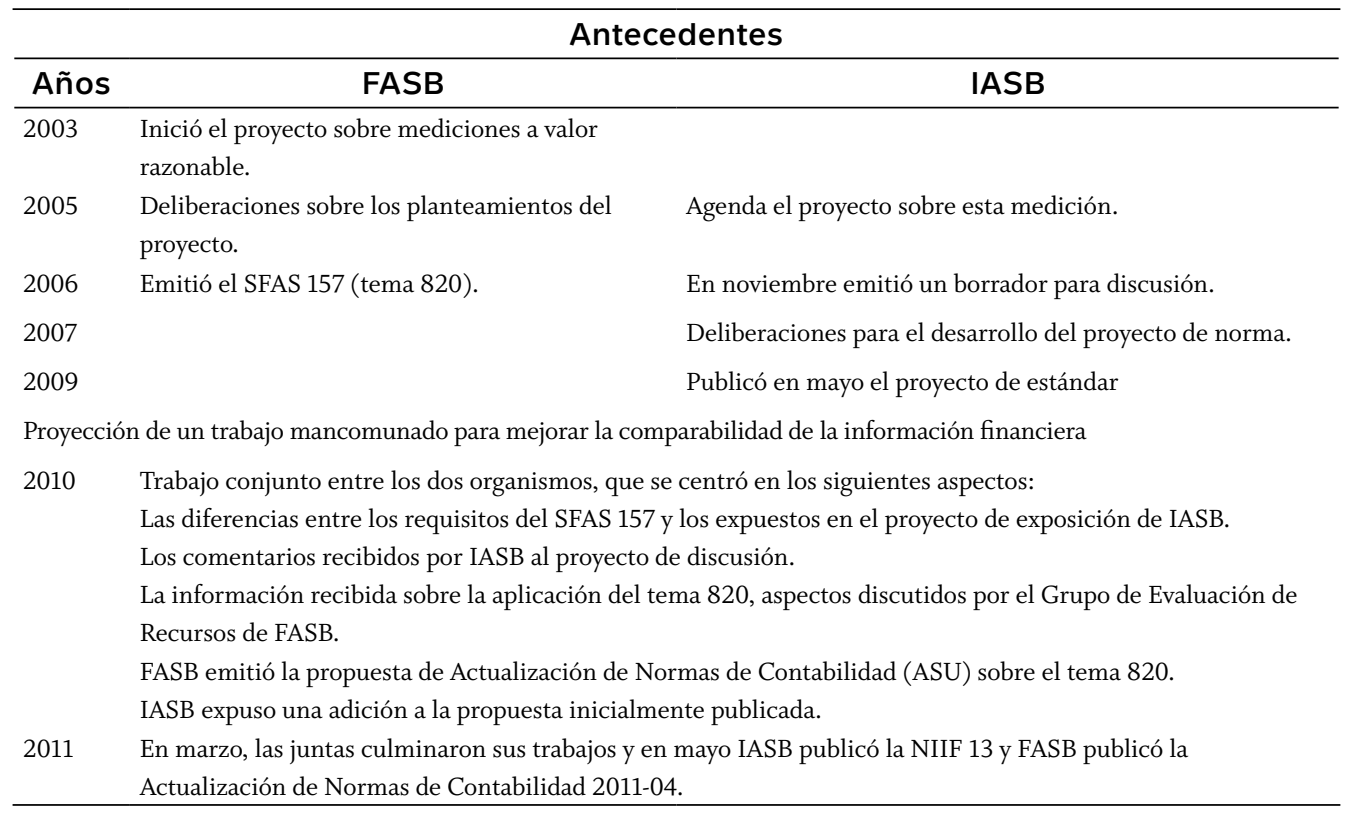

Tabla 1

Acciones conjuntas del FASB y el IASB para la emisión de la norma de valor razonable

Fuente: tabla elaborada por Martha Liliana Arias-Bello \& Édgar Emilio Salazar-Baquero (2011). NIIF 13: Mediciones del valor razonable

2 Es la condición actual de posmodernidad, cuando los simulacros no continúan asociados con ningún referente real y los símbolos, las imágenes y los modelos circulan, ajenos a cualquier objeto material real o ideal romántico. El concepto de hiperrealidad fue tomado por el grupo de Macintosh del trabajo de Jean Baudrillard. 


\section{2 / VOL. 16 / NO. 42 / SEPTIEMBRE-DICIEMBRE 2015}

El SFAS 157 tiene la intención de incrementar la consistencia, la comparabilidad y la transparencia de las mediciones hechas a valor razonable usadas en la información financiera.

La normatividad sobre medición a valor razonable, el SFAS 157, propuso el uso de inputs del nivel 1, 2 y 3, como técnicas de medición de activos y pasivos; el input se refiere a los supuestos que los participantes del mercado usarían en la determinación de precios, incluyendo los supuestos sobre riesgos inherentes en la técnica particular de valuación para medir el valor razonable, y los riesgos inherentes en los inputs para la técnica de valuación, que pueden ser observables y no observables.

Los inputs observables son los que reflejan los supuestos que utilizarían los participantes del mercado para fijar los precios de los activos o pasivos, con base en datos obtenidos en el mercado, a partir de fuentes independientes a la entidad que los reporta. Mientras los inputs no observables reflejan los propios supuestos que la entidad reporta, con base en los supuestos que los participantes del mercado usarían para fijar los precios de los activos y pasivos.

Según esta técnica de valuación, el valor razonable busca maximizar el uso de los inputs observables y minimizar el uso de los inputs no observables.

Mediante la emisión de la norma de valor razonable, el FASB obliga a las entidades a registrar sus activos y pasivos a valores de mercado, lo cual permite atender tres niveles diferentes como requisito en el cálculo de valor razonable definidos de la siguiente manera:

Las entradas del nivel 1 (SFAS 157, párrafo 24) son los precios cotizados (no ajustados) en un mercado activo para activos y pasivos idénticos a los cuales la entidad que reporta debe tener la capacidad de acceder. Dilucidando que un mercado activo es en el que concurren transacciones de activos y pasivos con frecuencia y volumen suficientes para ofrecer continuamente información sobre la fijación de precios. Considerando este precio como la evidencia más confiable del valor razonable, será usado para medir el valor razonable siempre y cuando esté disponible, excepto en los siguientes casos establecidos en los párrafos 25 y 26 :

- cuando la entidad que reporta tenga una gran cantidad de activos o pasivos similares que requiera medir a valor razonable, y cuando el precio del activo puede estar disponible en el mercado, pero no para cada uno de esos activos o pasivos individualmente. Esto da paso a que el valor razonable pueda ser medido empleando métodos alternativos de fijación de precios, y con ello la maniobra del valor de activos y pasivos registrados en los estados financieros. No obstante, muchas veces se hace imposible medir el valor razonable del activo, debido a que no hay un precio cotizado en un mercado activo para un activo idéntico o con las mismas características, que permita realizar una estimación fiable del precio que se podría obtener, por la venta del activo en una transacción realizada en condiciones de independencia mutua entre partes interesadas y debidamente informadas, hecho que crea mayor incertidumbre en la determinación del valor razonable, máxime cuando la misma normatividad 
permite que se maniobre el valor de los activos y pasivos de la empresa.

A tal efecto, Philip D. Bougen y Joni J. Young (2012), en su artículo Fair Value Accounting: Simulacra and Simulation [Contabilidad de valor razonable: simulacros y simulación], señalan que las propiedades que conforman la contabilidad de valor razonable son imaginarias, ya que los precios y los mercados son imaginarios, por no requerir necesariamente ser vistos para su aprobación o desaprobación. En tal sentido, los autores conceptúan que "nuestro interés en un imaginario o, más específicamente, cómo la realidad puede ser imaginada y re-imaginada" (p. 391).

Para hacer las anteriores afirmaciones, Philip D. Bougen y Joni J. Young (2012) se apoyan en las posturas de Jean Baudrillard (1983, p. 5) quien asevera que la "simulación amenaza la diferencia entre 'lo verdadero' y 'lo falso' entre 'lo real' y 'lo imaginario"', considerándose este el ambiente en el que se está moviendo la contabilidad de valor razonable, puesto que, bajo la concepción de simulacros, ahora la realidad contable es producida por "cálculos y supuesto del simulador, reformulación y recalibración para producir más simulacros y realidades" (Bougen \& Young, 2012, p. 392). Y lo veremos con mayor precisión en la explicación del nivel tres.

- Cuando el precio cotizado en el mercado activo puede no representar el valor razonable a la fecha de medición, pues el cierre del mercado fue antes de la fecha de medición. La entidad que reporta debe establecer y aplicar políticas consistentes para identificar esos eventos que puedan afectar las mediciones hechas a valor razonable. Pero si el precio cotizado se ajusta por nueva información, los ajustes hacen que la medición a valor razonable se ubique en el nivel más bajo.

Las entradas del nivel 2 (SFAS 157, párrafo 28). Estos inputs son diferentes a los precios cotizados, mencionados en el nivel 1, que son observables para el activo o pasivo de forma directa o indirecta. Si el activo o pasivo tiene un término específico (contractual), la entrada del nivel 2 tiene que ser observable.

Las entradas del nivel 2 requieren precios cotizados para activos y pasivos en un mercado activo o un mercado no activo para cotizar los precios de activos idénticos o similares. También las entradas que son observables para los activos y pasivos como la tasa de interés, las volatilidades, las velocidades de prepagos, las severidades de pérdidas, los riesgos de tasa, la tasa de incumplimiento en el pago, entre otros. Con respecto a los ajustes del nivel 2, estos dependen de los factores de localización y condiciones específicas para cada activo o pasivo, la extensión en la cual las entradas se relacionan con elementos que sean comparables, y el volumen y nivel de actividad en los mercados en los que se observan las entradas y se pueden convertir un ajuste para la medición a valor razonable en una medición de un nivel diferente.

Análogamente, conviene decir con respecto al nivel 2 que Philip D. Bougen y Joni J. Young (2012, p. 394) contemplan que el "grado y el tipo de ajustes requeridos en este nivel cambian 
la determinación de valor razonable hacia simulacros y simulación”. Porque "el estándar explícitamente reconoce la importancia de tasar el nivel de semejanzas, comparabilidad y desviación de un precio citado". Y con igual relevancia, el nivel de semejanzas y el grado de ajustes "podrían dar una medida de Nivel 3", reforzando el tema de la conectividad de nivel, ya que el Nivel 2 se fija relacionándose con el Nivel 3, el cual se analiza a continuación.

Las entradas del nivel 3 (SFAS 157, párrafo 30). Estas entradas son no observables y serán usadas para medir el valor razonable de activos y pasivos al no estar disponibles las entradas observables. Por tanto, las entradas no observables reflejan los supuestos o datos propios de la entidad que reporta, incluyendo los riesgos. Para ello, estas entidades no necesitan realizar esfuerzos para obtener información sobre los supuestos de los participantes del mercado. Los supuestos o datos propios de la entidad serán ajustados si la información está razonablemente disponible sin costos y esfuerzos indebidos que señalen que los participantes del mercado usarían supuestos diferentes.

Vemos cómo el estándar de valor razonable reconoce en el nivel 1 la necesidad de un mercado activo, en la que el activo o pasivo se pueda comparar con otro con las mismas características, para determinar su valor. Mientras en los niveles 2 y 3 notamos cómo la norma abre paso a entradas inobservables y con ello a juicios de valor en los que el preparador de los estados financieros puede crear, imaginar y organizar la información de acuerdo a su experiencia, visiones y beneficios, hasta encontrar la posibilidad de tomar las medidas de valor razonable que considere más convenientes, transgrediendo así muchas de las características básicas que debe contener la información financiera para que realmente sea útil y de calidad para la toma de decisiones de los diferentes usuarios.

Para que la información financiera logre el objetivo central de ser útil y de calidad debe contar con las características cualitativas de relevancia, materialidad, fiabilidad, pertinencia, comparabilidad, oportunidad, comprensibilidad, verificabilidad, puntualidad y representación fiel.

En el Marco conceptual para la información financiera (FASB, 2010; IASB, 2009), la calidad de la información financiera se logra con el cumplimiento de las características cualitativas de esta información. Al respecto, Álvaro Javier Romero-López (2007) afirma lo siguiente:

\begin{abstract}
Las características cualitativas de la información financiera son en estricto sentido los requisitos mínimos de calidad que debe contener cualquier estado financiero; pues si la información financiera está preparada con calidad le será útil al usuario en la toma de decisiones económicas (p. 97).
\end{abstract}

En este perfil se encuentran Gerardo Guajardo-Cantú y Nora E. Andrade de Guajardo (2008, p. 35) al aseverar que la calidad de la información financiera se obtiene al "cumplir con una serie de características que le dan valor. Estas características se consideran básicas, pues revisten la mayor importancia”.

Conviene resaltar que la calidad y la utilidad se alcanzan al cumplir, íntegramente, con 
todas las características cualitativas y, en ningún caso, alguna característica prevalecerá en detrimento de otra, es decir, todas tienen adherido el mismo nivel de importancia.

Con respecto a la comprensibilidad y a la relevancia cabe anotar lo siguiente:

Primero, la comprensibilidad está relacionada con el grado de conocimiento que posean los usuarios sobre las actividades que se desarrollan en el mundo de los negocios y sobre contabilidad, ya que no solo será suficiente con que una entidad presente información comprensible, sino que se hace imprescindible que el destinatario esté en capacidad de entenderla. Esta situación es ilustrada por Donald Kieso y Jerry J. Weygandt (2006) con el siguiente ejemplo:

Supongamos que la IBM Corp. publica un informe de ganancias de tres meses (informe entre ejercicios) con información pertinente y confiable para la toma de decisiones. Lamentablemente, ciertos usuarios no entienden el contenido y significado de esta información. De tal manera que, aunque la información sea muy pertinente y confiable, carece de utilidad para los usuarios que no la entienden (p. 38).

Segundo, para que la información financiera sea relevante en la toma de decisiones del usuario que la utiliza debe: a) servir de base en la elaboración de predicciones y en su confirmación (posibilidad de predicción y confirmación); y b) mostrar los aspectos más significativos de la entidad reconocidos contablemente (importancia relativa) (NIF, A-4, p. 20).
Ante esta afirmación, Inés Fortis-Pita e Inmaculada García-Gutiérrez (2006, p. 12) manifiestan que la información financiera cumplirá con la característica cualitativa de relevancia cuando "tiene capacidad para confirmar o modificar las expectativas de quienes toman decisiones económicas, porque esa información reduce la incertidumbre vinculada a cualquier decisión".

Es de acotar que el enunciado de relevancia contiene implícita la capacidad de la información contable para realizar predicciones o estimaciones relacionadas con aspectos de la situación financiera y el rendimiento futuro de la entidad, unido a esto, ahora la contabilidad debe tener la capacidad de garantizar que las estimaciones y predicciones realizadas ocurran.

De otra parte, una preocupación expresada en un comentario sobre el informe que presentó la Securities and Exchange Commission (SEC) de Estados Unidos, agencia federal encargada de la supervisión de los mercados financieros, en 2008 señaló: "las valoraciones de Nivel 3 del SFAS 157 llevan una connotación negativa inadecuada que podría ser evitada estableciendo estándares de valoración consecuentes para realzar la calidad de las valoraciones" de instrumentos financieros que son difíciles de valorar a la luz del nivel 3 (apéndices, A 16).

De lo expuesto, resulta pertinente explicar las posturas de Philip D. Bougen y Joni J. Young (2012), cuando plantean que el FASB debía reconocer que la utilización del nivel 3 para la valoración de instrumentos financieros requiere la simulación para hallar su valor razonable. Y que este hecho corresponde a la 


\section{6 / VOL. 16 / NO. 42 / SEPTIEMBRE-DICIEMBRE 2015}

creación de realidades más que al logro de una representación en contabilidad.

Y añaden:

Tal posición habría corrido el riesgo de violar el principio de neutralidad de la configuración estándar o diciendo las cosas como son. En este escenario, la autoridad de orientación FASB para los protocolos de medición ya sea enmarcada en términos de fidelidad representativa, fiabilidad o utilidad podría colapsar bajo el peso del reconocimiento de que la realidad contenida en los informes de contabilidad puede ser imaginada y vuelta a imaginar en una multitud de formas, por múltiples partes con diferentes objetivos (p. 392).

\section{Conclusiones}

El criterio de valoración de activos y pasivos a valor razonable, comúnmente utilizado en contabilidad como un mecanismo de medición, incidió de manera determinante en la crisis económica ocurrida en Estados Unidos en el sector inmobiliario en 2008, pues como consecuencia de la utilización de los niveles 2 y 3 de entradas inobservables en la determinación del valor razonable de los activos y pasivos, se produjo una alteración de los resultados presentados en los estados financieros, en vista de que estos registraban ganancias no realizadas que no correspondían a la realidad del sector inmobiliario para ese momento.

Además, tal situación se presentó, porque esas valoraciones no podían ser contrastadas en la realidad, por el hecho de no haberse realizado mediante la utilización de un mercado activo en que se dieran los supuestos que los participantes del mercado utilizarían para determinar los precios que recibirían tanto al vender un activo, como al transferir un pasivo, a partir de fuentes independientes a la entidad que reporta, lo que hace más objetiva la medición. Por el contrario, se utilizaron entradas no observables producto de mercados imperfectos e inactivos en los que la entidad que reporta utiliza supuestos internos para fijar los precios de sus activos y pasivos.

Con ello, los resultados presentados en los informes financieros no revelan la situación real de las organizaciones, lo que crea limitaciones para la toma de decisiones, aumenta los riesgos en la confirmación de las predicciones por parte de los usuarios y en el patrimonio de las empresas. Esto ocasiona que el objetivo central de la información financiera - brindar información útil a los diferentes usuarios alrededor del mundo para una adecuada toma de decisiones - no se cumpla, debido al incumplimiento de una de las características cualitativas que debe contener la información financiera como la de ser relevante.

Finalmente, es importante señalar que la aplicación de la contabilidad de valor razonable nos enseña que las valoraciones y los registros contables presentados en los estados financieros por los preparadores de la información financiera no se apartan de la subjetividad, pareceres, conveniencias, creaciones e imaginaciones unidas a la experiencia del preparador. 


\section{Referencias}

Álvarez-Álvarez, Harold (2009). La dicotomía valorativa en el modelo contable NICNIIF: una reflexión a la luz de la teoría económica del valor. Adversia, 4, 61-93. Disponible en: http://aprendeenlinea.udea. edu.co/revistas/index.php/adversia/article/ download/2033/1682

Arias-Bello, Martha Liliana \& Salazar-Baquero, Édgar Emilio, NIIF 13: Mediciones del valor razonable. Disponible en: http:// www.cpcpcolombia.org/documentos/niif/ MEDICION\%20VALOR\%20RAZONABLE. pdf

Baker, C. Richard (2009). ¿Cuál es el significado del "interés público"? Examinando la ideología de la profesión de la contaduría pública americana. En Mauricio Gómez-Villegas \& Carlos Mario Ospina-Zapata, Avances interdisciplinarios para una comprensión crítica de la contabilidad. Textos paradigmáticos de las corrientes heterodoxas, 223-239. Medellín: Universidad Nacional de Colombia, Universidad de Antioquia.

Baudrillard, Jean (1983). The Precession of Simulacra. En Simulations, 1-79. New York: Semiotext[e].

Bougen, Philip D. \& Young, Joni J. (2012). Fair Value Accounting: Simulacra and Simulation. Critical Perspectives on Accounting, 23 (4-5), 390-402.

Cabeza del Salvador, Ignacio (2009). Reflexiones sobre la crisis económica y el papel de la auditoría pública. Auditoría Pública: Revista de los Órganos Autónomos de Control Externo, 47, 27-46. Disponible en: http://www. auditoriapublica.com/hemeroteca/pag\%20 63\%20a\%2070.pdf

Castellanos-Sánchez, Heiberg Andrés (2010). El valor razonable y la calidad de la información financiera. Revista Visión Gerencial, 9 (2), 269-282. Disponible en: http://erevistas.saber.ula.ve/index.php/ visiongerencial/article/view/1055/1024

Delgado-Piña, Leonor (2011). El valor razonable en tiempos de crisis. Partida Doble, 22 (235), 74-81. Disponible en: http://pdfs. wke.es/9/0/2/4/pd0000069024.pdf

Financial Accounting Standards Board, FASB (2006). Fair Value Measurements. SFAS 157. September, I. Disponible en: http://www. fasb.org/cs/BlobServer?blobkey=id\&blobw here $=1175823288587 \&$ blobheader $=$ applic ation/pdf\&blobcol $=$ urldata\&blobtable $=\mathrm{Mu}$ ngoBlobs

Financial Accounting Standards Board, FASB (2006). NIF A-4, Características cualitativas de los estados financieros.

Financial Accounting Standards Board, FASB \& International Accounting Standards Board, IASB (2011). Marco conceptual. Disponible en www.ifrs.org

Fortis-Pita, Inés \& García-Gutiérrez, Inmaculada (2006). La contabilidad según valor razonable.

Guajardo-Cantú, Gerardo \& Andrade de Guajardo, Nora E. (2008). Contabilidad financiera. México: McGraw-Hill. Disponible en: http:// informacionfinanciera.weebly.com/ uploads/2/1/2/7/21272330/contabilidad_ financiera-1.pdf 
Herz, Robert (2006, March 29). Congressional Testimony: FASB Chairman Robert H. Herz Testifies before the Capital Markets, Insurance and Government Sponsored Enterprises Subcommittee of the Committee on Financial Services.

Herz, Robert H. (2009, March 12).

Congressional Testimony: FASB Chairman Robert H. Herz Testifies before the United States House of Representatives Financial Services Subcommittee on Capital Markets, Insurance, and Government Sponsored Entities. Disponible en: www.fasb.org, http:/www.accountingfoundation.org/ jsp/Foundation/Page/FAFSectionPage\&c id $=1351027547897$

Ijiri, Yuji (1975). Theory of Accounting Measurement. Serie Studies in Accounting Research, 10. Sarasota, Florida: American Accounting Association.

International Accounting Standards Board, IASB (2011). Marco conceptual para la información financiera. Comité de Revisión de la Fundación IFRS (traducción oficial).

International Accounting Standards Board, IASB (2014). Norma internacional de información financiera 13, medición del valor razonable. Londres, Inglaterra. Comité de Revisión de la Fundación IFRS (traducción oficial).

International Accounting Standards Board, IASB (2015). Norma internacional de información financiera para pequeñas y medianas entidades. Londres, Inglaterra. Madrid: CISSPRAXIS, traducción oficial al castellano.
Kieso, Donald \& Weygandt, Jerry J. (2006). Contabilidad intermedia. México: Limusa Wiley.

Littleton, Ananias Charles (1955). Prestige for Historical Cost [El prestigio del costo histórico]. Illinois CPA, Illinois Certified Public Accountant, 17, 23-27.

Macintosh, Norman B.; Shearer, Teri; Thornton, Daniel B. \& Welker, Michael (2000). Accounting as Simulacrum and Hyperreality: Perspectives on Income and Capital. Accounting, Organizations and Society, 25 (1), 13-50.

Maltby, Josephine (2000). The Origins of Prudence in Accounting. Critical Perspectives on Accounting, 11 (1), 51-70.

Mattessich, Richard (2002). Contabilidad y métodos analíticos. Buenos Aires: La Ley.

Menger, Carl (1871). Principios de economía política. Barcelona: Ediciones Orbis. Disponible en: http://www.eumed.net/cursecon/ textos/menger/

Romero-López, Álvaro Javier (2007). Principios de contabilidad. México: McGraw-Hill.

Sánchez-Salamanca, Lilia Beatriz (2009). Un paseo por la crisis económica mundial. Un recuento de la crisis y algunas moralejas de la crisis financiera internacional, hasta ahora. Economía Autónoma, 3.

Stojilković, Milorad (2011). Towards a Criticism of Fair Value Accounting. Facta Universitatis, Series: Economics and Organization, 8 (1), 91-109. Disponible en: http://facta.junis.ni.ac.rs/eao/eao201101/ eao201101-08.pdf

Viloria-Ortega, Norka (2013). Los retos de la contabilidad. Una visión desde los avances de la teoría contable. Revista Lúmina, 14, 
PERSPECTIVA CRÍtICA DEL VALOR RAZONABLE EN EL MARCO DE LA CRISIS FINANCIERA / S. PEREA / 779

40-52. Disponible en: http://www.umanizales.edu.co/publicaciones/campos/economicas/lumina/recursos/14/10.pdf

- Fecha de recepción: 25 de marzo de 2015

- Fecha de aceptación: 18 de abril de 2016

- Disponible en línea: 15 de mayo de 2016

\section{Para citar este artículo}

Perea-Murillo, S. P. (2015). Perspectiva crítica del valor razonable en el marco de la crisis financiera. Cuadernos de Contabilidad, 16(42), 761-779. http://dx.doi. org/10.11144/Javeriana.cc16-42.pcvr 
